\title{
SPRi-based hemagglutinin quantitative assay for influenza vaccine production monitoring.
}

\author{
Laurent Durous ${ }^{1,2}$, Thomas Julien ${ }^{2}$, Blandine Padey ${ }^{2}$, Aurélien Traversier ${ }^{2}$, Manuel Rosa-Calatrava ${ }^{2}$, \\ Loïc J. Blum ${ }^{1}$, Christophe A. Marquette ${ }^{1}$, Emma Petiot ${ }^{1,3}$ \\ ${ }^{1}$ Univ Lyon, GEMBAS Team, CNRS UMR 5246, INSA, CPE-Lyon, ICBMS, Université Claude Bernard Lyon 1, Villeur- \\ banne cedex, France \\ ${ }^{2}$ Univ Lyon, VirPath Team, International Center for Infectious diseases Research, Inserm U1111, CNRS UMR5308, ENS \\ Lyon, Université Claude Bernard Lyon 1, Faculté de Médecine RTH Laennec, LYON, France \\ ${ }^{3}$ CPE Lyon, Bâtiment Hubert Curien, 43 bd du 11 Novembre 1918, LyonTech - La Doua, Villeurbanne, France
}

\begin{abstract}
Influenza vaccine manufacturers lack tools, whatever the involved production bioprocess (egg or cell-based), to precisely and accurately evaluate vaccine antigen content from samples. Indeed, single-radial immunodiffusion (SRID) assay remains the only validated assay for the evaluation of influenza vaccine potency. We hereby report an imaging surface plasmon resonance (SPRi) biosensor for the quantification of both split vaccine antigen and influenza virus particles from egg- and cell-based production samples, respectively. The biosensor, based on the fetuin-hemagglutinin interaction, presents higher reproducibility $(<3 \%)$ and a greater analytical range $(0.03-20 \mu \mathrm{g} / \mathrm{ml})$ than SRID for bulk monovalent and trivalent vaccine and its limit of detection was evaluated to be 100-time lower than the SRID's one. Finally, viral particles production through cell culture-based bioprocess was also successfully monitored using the developed assay and a clear correlation was found between the biosensor response and the sample virus particle content.
\end{abstract}

\section{INTRODUCTION}

Current gold standard influenza vaccine production is based on egg inoculation technology which is every year endangered by the threat of a new pandemic outbreak. Alternative production methods based on standardized cell culture processes have been successfully proposed and it is believed to be the future of the influenza vaccine production since it should be standardized, monitored and controlled, like other bio-production processes. Nevertheless, when it comes to optimization and monitoring of vaccine production processes, one of the most interesting parameters to follow is the virus particles content, directly in the bioreactor. Unfortunately, accessible methods are not useful for on-line monitoring, while WHO and regulatory agencies are strongly supporting the development of improved influenza vaccine potency assays ${ }^{1,2}$. Presently, influenza vaccine lots release relies on the standard singleradial immunodiffusion (SRID) assay ${ }^{1}$. SRID requires strainspecific reference sera and antigen reagents to determine the amount of influenza hemagglutinin (HA) antigen. The annual preparation of these reagents takes up to 6 months and constitutes critical bottleneck for the release of vaccine lots ${ }^{3-5}$.

In order to push forward the development and optimization of cell culture-based virus production, rapid, specific, sensitive and in-process quantification tools need to be developed. This will enable to precisely follow the production level and optimize the bioprocess operating conditions.

A large set of analytical technologies has been evaluated to replace or complement SRID in early phases of process developments. Various immunoassays including ELISA, latex bead agglutination or slot-blot were developed with either monovalent antibodies or reference sera to target influenza antigens ${ }^{6-8}$. Alternative chromatography-based strategies, like RP-HPLC and LC-MS, were also proposed to directly quantify HA content in vaccine samples ${ }^{9,10}$. Regarding in-process quantification of influenza viruses, physical characterization of the viral particles through Dynamic Light Scattering (DLS), Tuneable
Resistive Pulse Sensing (TRPS), Flow Virus counter and FFFMALS were proposed ${ }^{11}$. Although these techniques present several advantages such as reduced analysis time (minuteshours vs. days) and increased reproducibility, their weaknesses stand on a low specificity and on the absence of information regarding HA bioactivity. We are proposing herein the use of imaging Surface Plasmon Resonance (SPRi), which represents an interesting alternative being able of real time detection of specific biomolecular interactions with hemagglutinin, with a potential to be on-line integrated to the production process via incorporated fluidic system.

SPR is commonly used to detect biomolecular interactions and has also been extensively used to determine affinity constants of several different influenza hemagglutinins toward a large panel of ligands. However, while SPR has already proven its potential for label-free quantitative evaluation of bioactive antibodies and recombinant proteins produced in cell culture ${ }^{12,13}$, very few studies were performed on the use of SPR-based assay for quantitative evaluation of influenza hemagglutinin antigens or virus particles ${ }^{4,12}$. The present work is dedicated to the development of a SPRi-based assay for the rapid and label-free quantification of both influenza virus particles and hemagglutinin content in influenza vaccine. Based on immobilized fetuin, a generic sialic acid-containing ligand of influenza hemagglutinin antigens, our quantitative assay harbored specificity and reproducibility with both influenza split vaccine and virus particles produced from egg- and cell-based systems. Moreover, particular attention has been given to the regeneration capability of the biosensor surface, leading to further on-line real-time monitoring and development for cell-based bioprocesses. 


\section{MATERIEL AND METHODS}

\section{Reagents}

Fetuin from fetal bovine serum, bovine serum albumin (BSA), ethyl dimethyl carbodiimide (EDC), Nhydroxysuccinimide (NHS), sodium dodecyl sulfate (SDS), polysorbate 20 , triton-X100, bacterial neuraminidase from $C$. perfringens and trypsin acetylated from bovine pancreas were purchased from Sigma (Saint Quentin Fallavier, France). Sodium acetate buffer and ethanolamine-HCL were acquired from Sierra sensors (Hamburg, Germany). Zwittergent 3-14 and Phosphate Buffer Saline (PBS) tablets were acquired from Merck (Darmstadt, Germany). All solutions were prepared using milliQ water. Oseltamivir carboxylate was from Roche (Bale, Switzerland). Trivalent inactivated vaccine were purchased from Sanofi Pasteur (Vaxigrip® 2016/2017, lot \#N2A381M, Sanofi Pasteur, Lyon, France) and recombinant A/California/04/2009 hemagglutinin from Sinobiological (Beijing, China). Madin-Darby canine kidney cells (MDCK, ATCC CCL34), serum free Ultra-MDCK and EMEM media, L-glutamine and penicillin/streptomycin solutions were from Lonza (Amboise, France). Optipro, SFM4Transfx-293 and 2X MEM media, Nobel agar, Coomassie blue and BCA assay reagents were from Thermo Fisher (Dardilly, France). Biobeads SM2 were purchased from Bio-RAD (Les Ulis, France). SRID standard antigen (NIBSC 09/196 and NIBSC 12/214) and polyclonal serum (NIBSC 14/134) for H1N1 A/California/7/2009 and H3N2 A/Victoria/361/2011 were obtained from NIBSC (http://www.nibsc.org).

\section{SPR imaging system}

SPR imaging experiments were performed using a SPR-2 instrument (Sierra Sensors, Hamburg, Germany). The sensor is based on Kretschmann configuration and consists in a microfluidic flow cell and a gold-coated prism functionalized with carboxylic acid groups (Amine coupling sensor, Sierra Sensors) useful for ligand immobilization. Biomolecular interactions at the surface of the sensor leads to changes in local refractive. Recorded images of the flow cell are processed in order to obtain a numerical signal and generate sensorgrams expressed in response unit [RU].

Raw SPR data (in RU) were processed through a custom MATLAB (The Mathworks, Natick, USA) script for interpolating and smoothing/despiking. Sensorgrams were obtained by first subtracting the signal from the reference surface to the signal from sensing surface for each sample in order to obtain standardized data. Initial response rates $\left(\left[R U . s^{-1}\right]\right)$ were calculated based on the slope of the referenced sensorgrams for the first 30 seconds of the association phase, while response level was determined as the maximum response at the end of the association phase.

The limits of detection (LOD) and calibration (LOQ) were subsequently determined based on the following equations:

$$
L O D=3.3 \frac{\sigma}{\text { slope }} ; L O Q=10 \frac{\sigma}{\text { slope }}
$$

where $\sigma$ was the standard deviation of 3 consecutive injections of negative sample $(0 \mu \mathrm{g} \mathrm{HA} / \mathrm{mL})$. Statistical analyses were performed using GraphPad Prism 7 software (GraphPad Software, Inc., La Jolla, CA).

\section{Proteins' immobilization}

For immobilization purpose, proteins were used in a concentration of $200 \mu \mathrm{g} / \mathrm{mL}$ in $10 \mu \mathrm{mM}$ sodium acetate buffer, $\mathrm{pH}=4.5$. Immobilization protocol was performed directly within the flow cell of the SPR-2 apparatus. Briefly, a surface activation solution composed of $0.2 \mathrm{M}$ EDC and $0.05 \mathrm{M}$ NHS was applied at a flow rate of $25 \mu \mathrm{L} / \mathrm{min}$ on the surface of a SPRi sensor for $4 \mathrm{~min}$. Then, fetuin immobilization solution was injected and applied for 4 minutes. In parallel, a separate surface of the same sensor (see Figure 1-B) was functionalized with control proteins, either BSA or truncated fetuin (without terminal sialic acid moiety). Remaining activated carboxylic acid functions of the sensor surface were then deactivated using $1 \mathrm{M}$ ethanolamine injection for 3 minutes.

\section{Protein surface density}

According to the average density of proteins (i.e. $\rho_{\text {prot }} \approx 1.35$ $\mathrm{x} 10^{-21} \mathrm{~g} \cdot \mathrm{nm}^{-3}$ ) the minimum volume of a globular protein can be calculated based on the following formula ${ }^{14}$ :

$V_{\text {prot }}\left[\mathrm{nm}^{3}\right]=M W_{\text {prot }} /\left(\rho_{\text {prot }} \times N_{A}\right)$, where $\mathrm{N}_{\mathrm{A}}$ being Avogadro number.

Thus, assuming fetuin can be modeled as a globular protein of $48.4 \mathrm{kDa}$, calculated $V_{\text {fetuin }}$ is $59.5 \mathrm{~nm}^{3}$ and fetuin diameter $4.8 \mathrm{~nm}$, leading to a maximum theoretical fetuin surface density of $5.43 \times 10^{10}$ molecule $/ \mathrm{mm}^{2}$. Similarly, assuming bovine serum albumin can be modeled as a globular protein of 66 $\mathrm{kDa}$, calculated $V_{\text {fetuin }}$ is $81 \mathrm{~nm}^{3}$ and fetuin diameter $5.3 \mathrm{~nm}$, leading to a maximum theoretical BSA surface density of 4.42 $\mathrm{x} 10^{10}$ molecule $/ \mathrm{mm}^{2}$.

The mean SPRi signal obtained during fetuin and BSA immobilization were $1340 \mathrm{RU}$ and $868 \mathrm{RU}$, respectively. The measured values of reflectivity variation can be converted into protein surface density using the classical correspondence ${ }^{15}$ where 1 SPR unit corresponds approximately $1 \mathrm{pg} / \mathrm{mm}^{2}$. Surface densities of $1.66 \times 10^{10}$ molecule $/ \mathrm{mm}^{2}$ and $7.9 \times 10^{9} \mathrm{~mol}-$ ecule $/ \mathrm{mm}^{2}$ were calculated for fetuin and BSA, respectively.

\section{SPRi assay}

Running buffer for all experiments was composed of PBS containing $0.05 \%(\mathrm{v} / \mathrm{v})$ of Tween 20 . A constant running buffer flow rate of $25 \mu \mathrm{L} / \mathrm{min}$ was used during all experiments while temperature in the measurement chamber was maintained at $15^{\circ} \mathrm{C}$. Sample injection flow rate was fixed to 5 $\mu \mathrm{L} / \mathrm{min}$ for 6 minutes, leading to the injection of $30 \mu \mathrm{L}$ of each sample. Regeneration of the biosensor surface was performed through the injection, at a flow rate of $25 \mu \mathrm{L} / \mathrm{min}$, of $25 \mu \mathrm{L}$ of PBS containing $0.25 \%(\mathrm{v} / \mathrm{v})$ of sodium dodecyl sulfate.

The neuraminidase inhibitor oseltamivir carboxylate was added to all samples at a concentration of $10 \mu \mathrm{M}$, to prevent cleavage of sialic acid residues from the fetuin modified sensor.

\section{Preparation of split inactivated virus from ovoculture}

Pandemic influenza viral strain NYMC X-179A (reassortant A/H1N1/California/07/2009, NIBSC 09/216) was used for the production of split inactivated virus. A stock production of Triton-fragmented $\mathrm{H} 1 \mathrm{~N} 1$ influenza virus was realized by ovoculture on 11-day-old embryonated hen's eggs. Allantoic fluid was harvested 72 hours post infection, concentrated by ultracentrifugation (UC) and purified through $25-60 \%$ sucrose gradient UC. Visible virus band was harvested and layered onto PBS buffer before a final UC. Resuspended pellets were fragmented with $0.5 \%$ Triton-X100 detergent for 1 hour at 
$37^{\circ} \mathrm{C}$ before being dialyzed against PBS. Removal of detergent was achieved thanks to Bio-beads SM2 resin. After beads removal, samples were inactivated with $0.01 \%$ formaldehyde for $72 \mathrm{~h}$ at $20^{\circ} \mathrm{C}$. This preparation was calibrated using SRID assay and constituted our reference samples. Split inactivated virus stored at $2-8^{\circ} \mathrm{C}$ remain stable for several months ${ }^{16}$.

\section{Preparation of influenza virus from cell culture}

Cell-based production of influenza virus was performed using Madin-Darby Canine Kidney cells (MDCK Lonza, ATCC CCL34). Cells were grown inT-175 T-flasks in serum-free Ultra-MDCK medium supplemented with L-glutamine and penicillin/streptomycin until reaching $70-80 \%$ confluency (approximately $115000 \mathrm{cell} / \mathrm{cm}^{2}$ ). For infection, influenza viruses from $\mathrm{H} 1 \mathrm{~N} 1$ pandemic NYMC X-179A strain (reassortant A/H1N1/California/07/2009, NIBSC 09/216)) were inoculated at a multiplicity of infection (MOI) of $10^{-4}$ virus/cell. Infection was performed in serum-free EMEM medium supplemented with L-glutamine, penicillin/streptomycin at a concentration of $100 \mathrm{U} / \mathrm{ml}$ and $1 \mu \mathrm{g} / \mathrm{ml}$ Trypsin. Cell cultures were harvested and clarified by 10 minutes low-speed centrifugation at $2000 \mathrm{~g}$ before being used for SPRi detection. Control cell cultures were obtained using identical protocol except for virus infection.

For the evaluation of viral production kinetics, cultures supernatant were sampled daily, clarified by 10 minutes lowspeed centrifugation at $2000 \mathrm{~g}$ and stored at $-80^{\circ} \mathrm{C}$ before analysis.

Titration of infectious viral particles (IVP) by plaque forming unit assay

Infectious titer $(\mathrm{IVP} / \mathrm{ml})$ of influenza virus particles were quantified using the tissue culture infectious dose at $50 \%$ $\left(\mathrm{TCID}_{50}\right)$ and plaque forming unit (PFU) assays. For plaque assay, confluent MDCK cells were plated in 6-well tissue culture plates, inoculated with $800 \mu \mathrm{l}$ of cell culture supernatant serially diluted (1:10) in EMEM medium, and incubated 1 hour at $37^{\circ} \mathrm{C}$ under continuous shaking. Cells were washed with EMEM medium and overlaid with $1.1 \%$ Nobel agar previously mixed 1:1 with 2 x MEM containing in addition 200 $\mathrm{U} / \mathrm{ml}$ of penicillin-streptomycin and $2 \mu \mathrm{g} / \mathrm{ml}$ Trypsin. The plates were then incubated for 3 days at $37^{\circ} \mathrm{C}, 5 \% \mathrm{CO}_{2}$ before visual examination of cytopathic effect.

\section{Quantification of total viral particles (VP)}

Total particles were quantified by Tunable resistive pulse sensing technique (TRPS) using qNano Gold (IZON Science, Lyon, France). Analyses were performed using TPU mem-

Table 1 : Detailed description of the different influenza samples used in the present study. * TIV: Trivalent Inactivated Vaccine. $\dagger$ HA content of influenza vaccine based on vaccine SRID titer provided by the manufacturer. $\$$ HA content provided by NIBSC.

\begin{tabular}{|c|c|c|c|c|}
\hline \multirow[b]{2}{*}{ Technique } & TIV Vaccine* & \multirow{2}{*}{$\begin{array}{l}\text { Split-inactivated H1N1 } \\
\text { virus } \\
\text { H1N1 X-179A produced by } \\
\text { ovoculture, purified and } \\
\text { fragmented }\end{array}$} & \multirow{2}{*}{$\begin{array}{l}\text { NIBSC influenza } \\
\text { reference reagents } \\
\text { Partially purified, inac- } \\
\text { tivated, freeze-dried } \\
\text { virus }\end{array}$} & \multirow{2}{*}{$\begin{array}{l}\text { H1N1 virus } \\
\text { H1N1 X-179A produced in } \\
\text { MDCK cell culture }\end{array}$} \\
\hline & $\begin{array}{l}\text { Trivalent, purified, } \\
\text { inactivated and frag- } \\
\text { mented virus }\end{array}$ & & & \\
\hline \multirow{2}{*}{ SRID $\left(\mu \mathrm{g}\right.$ HA.mL $\left.L^{-1}\right)$} & \multirow{2}{*}{$90^{\dagger}$} & \multirow{2}{*}{239} & $37(\mathrm{H} 1 \mathrm{~N} 1)^{\ddagger}$ & \multirow{2}{*}{-} \\
\hline & & & $79(\mathrm{H} 3 \mathrm{~N} 2)^{\ddagger}$ & \\
\hline $\begin{array}{l}\text { Neuraminidase assay } \\
(\mathrm{nmol} 4-\mathrm{Me} / \mathrm{h} / \mathrm{ml})\end{array}$ & 900 & 3000 & - & 80 \\
\hline $\begin{array}{l}\text { Total Particles } \\
\left(\text { particles.mL }{ }^{-1}\right)\end{array}$ & $1.15 \times 10^{11}$ & $1.58 \times 10^{12}$ & - & $1.71 \times 10^{10}$ \\
\hline
\end{tabular}

branes with a tunable nanopore of $150 \mathrm{~nm}$ (NP150). Prior analyses, samples were diluted at 1/5 in PBS buffer.

Quantification of Hemagglutinin (HA) antigen content and activity

HA concentration in split-inactivated lot of H1N1 virus has been determined by Single Radial Immunodiffusion assay (SRID) according to standard protocol with calibrated standard antigen (NIBSC 09/196) and polyclonal serum (NIBSC 14/134) of A/California/7/2009 ${ }^{17}$. Standard and samples were incubated with a final concentration of $1 \%$ Zwittergent 3-14 for 30 minutes at room temperature, diluted at $1: 1 ; 3: 4 ; 1: 2$; $1: 4$ and loaded in triplicate into wells. Gels were incubated for 20 hours at $22^{\circ} \mathrm{C}$ to enable antigen migration, then washed with PBS, dried and stained in Coomassie blue. Precipitating rings were measured using ProtoCol 3 (SYNBIOSIS), and HA content was determined in $\mu \mathrm{g} / \mathrm{mL}$ with the parallel line bioassay method by comparison of obtained dose-response curves of the samples with those of the standard antigen.

\section{RESULTS AND DISCUSSION}

The present study aims at developing and validating the use of a SPRi-based assay for the characterization of influenza virus production (egg or cell-based process). In order to fully validate the developed analytical system, various samples have been collected or prepared and characterized. A description of these samples is presented in Erreur ! Source du renvoi introuvable. together with their characterization methods and corresponding characteristics. Three complementary characterizations were performed on these samples: HA content (SRID), neuraminidase activity and total particles counting. Within the present study, commercial TIV vaccine (equally composed of antigens from $\mathrm{H} 1 \mathrm{~N} 1, \mathrm{H} 3 \mathrm{~N} 2$ and $\mathrm{B}$ strains) was used as a standard while split-inactivated and whole virus were used as bioprocess derived products issued from egg and cell-based production, respectively.

\section{SPRi assay optimization}

The main steps toward the development of an effective SPRi assay is the optimization of sensing and control layers, but also the study of the possible regeneration of these surfaces and their operational lifetime. The sensing layer chosen in the present study is a covalently immobilized fetuin glycoprotein bearing oligosaccharides with $\alpha-2,3-$ and $\alpha$-2,6-linked terminal sialic acid residues. 
Two different negative control layers were evaluated, one composed of immobilized albumin from bovine serum (BSA) and one composed of fetuin treated with bacterial neuraminidase allowing for sialic acid $-2,3, \alpha-2,6$ or $\alpha-2,8$ terminal link cleavage. This second control surface corresponds to a cropped fetuin structure, close to the asialofetuin structure, known for inducing no specific binding of HA ${ }^{18-20}$. Both sensing and control surfaces are present in a single flow injection cell (Figure 1-A).

These three surfaces were prepared through the covalent grafting of each protein via amide bound formation between the gold chip surface modified with carboxylic acid groups (thioctic acid) and available primary amino groups of the proteins. Fetuin grafting $\mathrm{pH}$ conditions were optimized to reach a maximal amount of fetuin and NANA residues available for hemagglutinin binding. Indeed, protein grafting on surface in flow conditions requires a contact between the surface and the protein, which is highly dependent upon the isoelectric point of the protein and then the $\mathrm{pH}$ of the immobilization solution. An optimum fetuin surface coverage of 1.66 $\mathrm{x} 10^{10}$ molecule $/ \mathrm{mm}^{2}$ was found using a grafting $\mathrm{pH}$ of 4.5 . This coverage compares well with the theoretical maximum coverage of $5.43 \times 10^{10}$ molecule $/ \mathrm{mm}^{2}$ calculated using protein size and comparing with immobilization SPRi signal (see Methods for calculation).

Specificity of the assay was first assessed using monomeric recombinant HA (H1N1 A/California/04/2009). No specific interaction was observed when the protein was injected through the biosensor (data not show). Our results were in accordance with previous studies ${ }^{4,21}$ and confirm the binding to sialic acid residues only of the bioactive trimeric form of HA antigen ${ }^{19,22}$, thus validating our fetuin-based SPR assay that aims to detect functional hemagglutinin. In this context, commercial Trivalent Inactivated Vaccine (TIV, Vaxigrip ${ }^{\circledR}$ 2016-2017, presented in Table 1) was used as a calibrated standard of bioactive HA antigen to determine the performance of the SPRi biosensor assay.

Figure 1-B depicted the biosensor responses when TIV vaccine $(10 \mu \mathrm{g} \mathrm{HA} / \mathrm{mL})$ was bring into contact with the different surfaces. As expected, BSA control surface is not able to hinder the non-specific interactions with the injected sample, leading to a poor specific to non-specific signal ratio of 6.4. On the contrary, cropped fetuin functionalized surface was shown to be a more reliable control surface, leading to a nonspecific signal ratio of 41 . Moreover, the observed interactions between sensing surface and influenza purified vaccine antigens was proven to specifically due to HA interactions with fetuin glycans.

Regeneration solution composition was also optimized to achieve a complete releasing, between each sequential injection, of the bound HA. Classically proposed regeneration solutions ${ }^{23}$ such as glycine $0.1 \mathrm{M} \mathrm{pH} 2, \mathrm{HCl} 10 \mathrm{mM}$ or $100 \mathrm{mM}, \mathrm{NaCl} 1 \mathrm{M}$ and $0.25 \%$ (v/v) SDS in PBS were then evaluated. The best results were obtained using injection of 25 $\mu \mathrm{L}$ of a regeneration solution composed of $0.25 \%(\mathrm{v} / \mathrm{v})$ SDS in PBS (Supporting Information 1). Example of the signal recovery is presented in Figure 2-A where SPRi baseline was recovered between each of 5 successive injections of TIV vaccine antigen $(10 \mu \mathrm{g} / \mathrm{HA} \mathrm{mL})$.
Assay lifetime was also assessed through 20 successive cycles of TIV vaccine antigen $(10 \mu \mathrm{g} \mathrm{HA} / \mathrm{mL})$ injection and consecutive regeneration. As depicted in Figure 2-B, a linear decrease of the biosensor response was observed (slope $=-9.7$ $\mathrm{RU} /$ injection; $\mathrm{R}^{2}=0.8685$ ). This decrease was attributed to a surface degradation during regeneration rather than to a sialic acid hydrolysis by the virus neuraminidase, as the neuraminidase inhibitor oseltamivir carboxylate was systematically added to each sample. A correction factor was then applied to all subsequent experiments, adjusting the measured biosensor response using the calculated decrease slope.

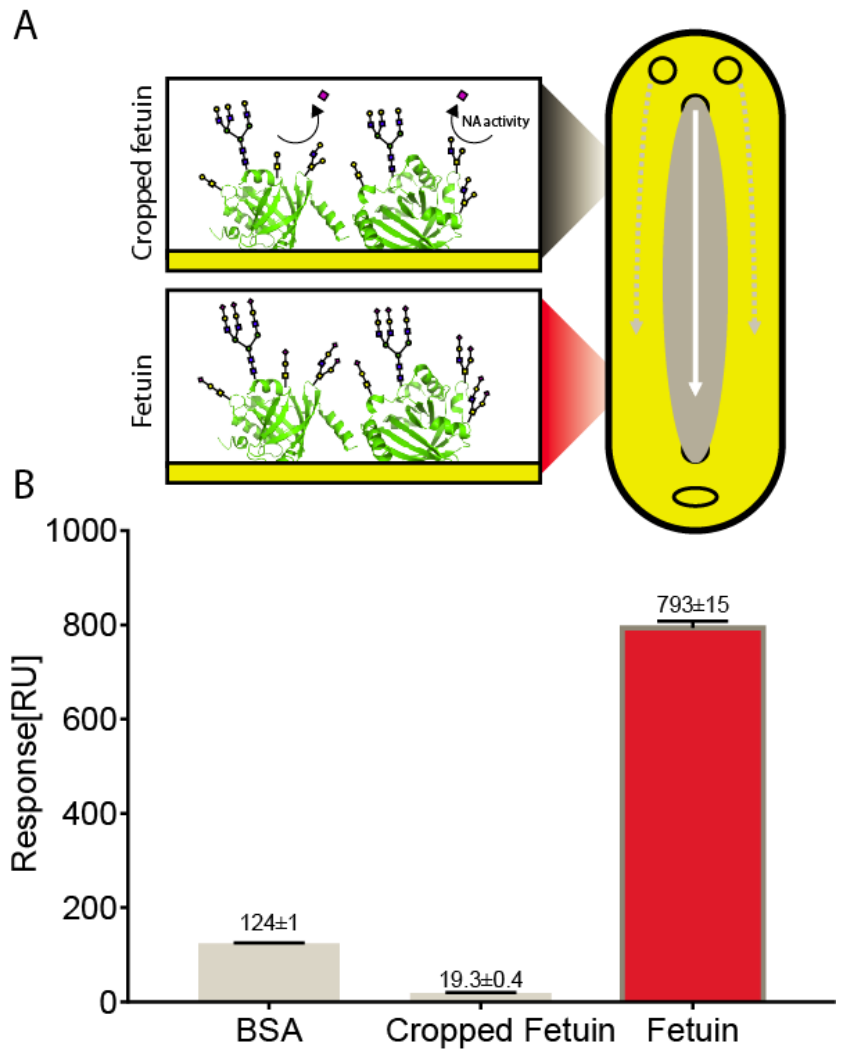

Figure 1 Control and sensing SPRi surfaces. A: Schematic representation of the two distinct measurement zone of the SPRi biosensor flow cell: control (grey) and sensing (red) layers; white arrow: analyte flow; grey arrows: buffer flow. Glycan structures on fetuin (PDB 3APX) according to published data ${ }^{24}$. Terminal sialic acid moieties (purple diamond) are removed by bacterial neuraminidase (NA) in-situ before processing samples. B: SPRi responses obtained for control and sensing surfaces with injected TIV vaccine $(10 \mu \mathrm{g} \mathrm{HA} / \mathrm{mL})$.

Finally, reproducibility of the assay was evaluated (Figure 2-C). The relative standard deviation (RSD) of the response was calculated on the basis of 7 consecutive injections of TIV vaccine antigen $(10 \mu \mathrm{g} \mathrm{HA} / \mathrm{mL})$ and regeneration. The high reproducibility of our assay was then demonstrated with RSD of $3 \%$ and $1.3 \%$ calculated based on initial binding rate and response level, respectively. As a comparison, relative standard deviation of the classic SRID assay for influenza HA quantification is at best $6 \%$, while the average RSD of 
Vaxarray sandwich immunoassay system has been estimated to $8 \%{ }^{4,6}$. Thus, after having validated the specificity of our regenerable sensor toward HA antigen and its excellent repro-

A.

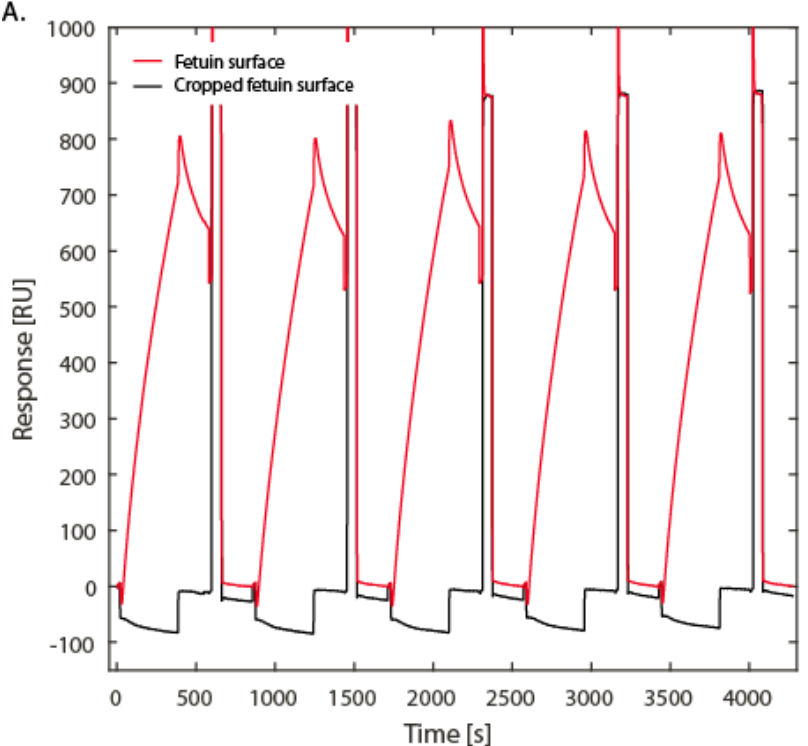

ducibility, we further evaluated its sensitivity and applicability for sensing hemagglutinin obtained from different influenza strains.

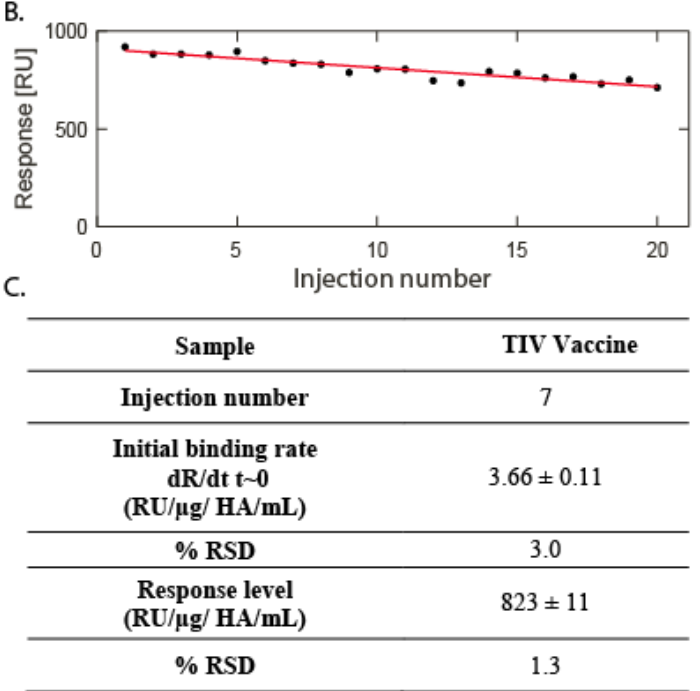

Figure 2 Biosensor reproducibility, stability and regeneration. A: Biosensor raw data obtained following 5 consecutive injections of TIV vaccine antigen $(10 \mu \mathrm{g} \mathrm{HA} / \mathrm{mL})$ with consecutive regeneration. B: Operational stability of the fetuin sensing layer toward multiple regenerations using $0.25 \%(\mathrm{v} / \mathrm{v})$ SDS in PBS. C: Reproducibility of the biosensor signal following several injections of TIV vaccine anti-

gen samples

\section{SPRi biosensor performances}

In order to evaluate the sensitivity of our biosensor for hemagglutinin-containing samples, sequential injections of vaccine dilutions were performed, the SPRi signal quantified and the corresponding calibration curve built (Figure 3-A). Linear dynamic range of the assay together with its limit of detection and limit of quantification are presented in Figure 3C. These parameters were determined using both the initial binding rate of the biosensor (RU/s) and the biosensor response (RU). Linearity of the biosensor response was shown to be optimum when the analytical parameter used to build the calibration curve was the initial binding rate. Indeed, linear regression parameters of $\mathrm{R}^{2}=0.9998$ and $\mathrm{R}^{2}=0.9778$ were calculated for initial binding rate and response, respectively. The assay was also shown to offer a large dynamic range with a linear response over three orders of magnitude, between 0.03 and $20 \mu \mathrm{g} \mathrm{HA} / \mathrm{ml}$. These results shall be compared to the dynamic range commonly obtained for SRID validated assay, i.e. between 3 and $36 \mu \mathrm{g} \mathrm{HA} / \mathrm{ml}^{25}$, demonstrating the superiority of the presently developed fetuin-based SPRi which offers a 100-time higher sensitivity than conventional SRID potency assay and to quantify samples with lower HA antigen content. These results open the path to evaluation of influenza vaccine production consistency and robustness in-process with the quantification of HA antigens along the production and purification. It is also a new technology potentially allowing the evaluation of HA antigen content of new cell-based or VLP-based influenza vaccine under development. Indeed, such processes generally display lower HA concentrations within the production phases, especially in the upstream production steps.

We further evaluated the biosensor performances by comparing the response obtained in the presence of two bioactive forms of HA produced in egg: i) TIV vaccine, equally composed of antigens from $\mathrm{H} 1 \mathrm{~N} 1, \mathrm{H} 3 \mathrm{~N} 2$ and B strains ${ }^{26}$, and ii)
$(10$

Split-inactivated H1N1 influenza virus. Sensorgrams and corresponding association and dissociation constants calculated using the classical 1:1 association-dissociation model (global fitting in kinetic-limited conditions) are given in Supporting Information 2. As can be seen, TIV vaccine interaction with the immobilized fetuin evidenced a much higher dissociation constant than the freshly split $\mathrm{H} 1 \mathrm{~N} 1$ virus sample. This affinity decrease was documented and characterized previously by Kuck and co-workers and Khurana et al. ${ }^{4,6}$, and was attributed mainly to differences in quaternary structures between HA antigens and in the degree of chemical modifications between reference reagents and vaccine samples. Thus, the present biosensor also has the potentiality to deeply characterize vaccine virus production and a complementary study was performed about the interaction of $\mathrm{H} 1 \mathrm{~N} 1$ and $\mathrm{H} 3 \mathrm{~N} 2$ viruses with the present biosensor. NIBSC reference reagents for $\mathrm{H} 1 \mathrm{~N} 1$ and H3N2 strains were injected in the biosensor and the corresponding signals recorded. Results are presented in Figure 3-B. One more time, the biosensor was able to characterize interactions between immobilized fetuin and HA from two different strains with clear variations in kinetic behaviors.

\section{SPRi detection of cell-based bioprocess samples}

One of the aims of the present study being the demonstration of the biosensor's capacity to follow virus production from various bioprocesses, detection within samples obtained from cell-based process was evaluated. First, various culture media in use for cell-based processes described in the literature $^{27,28}$ (EMEM, Ultra-MDCK, Optipro and SFM4TransFX) were studied according to non-specific signal triggering. Figure 4 presents the obtained signals in the presence of the different culture media.

These results demonstrate that among the four tested media, three of them, EMEM and Ultra-MDCK used for adherent MDCK cell culture and Optipro used for Duck-T17® avian 
A.

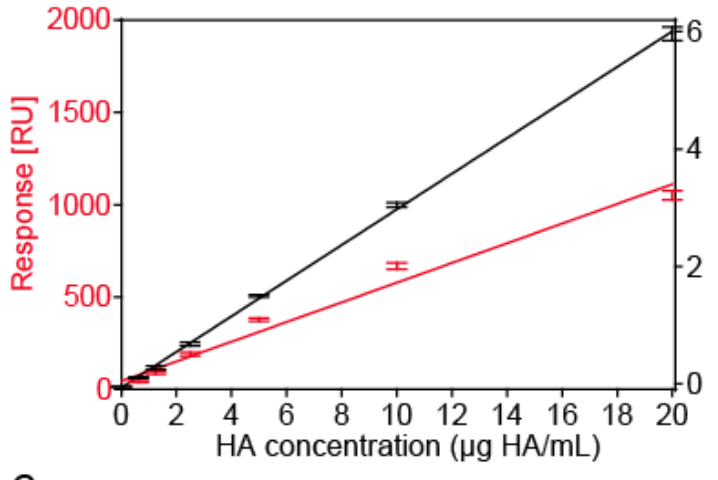

C.
B.

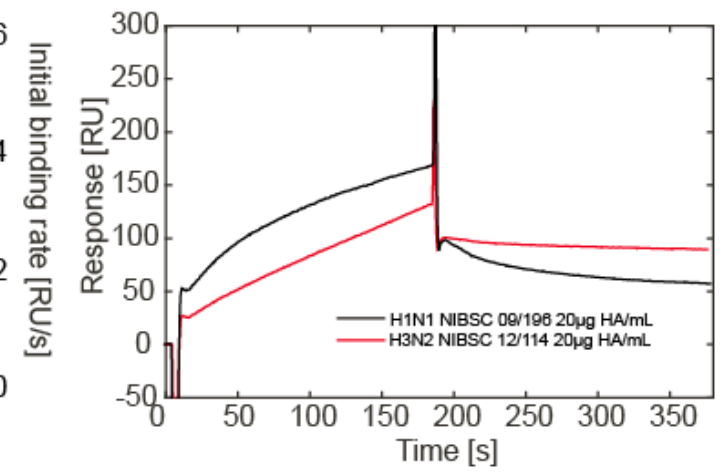

\begin{tabular}{|c|c|}
\hline Parameters & TIV Vaccine \\
\hline Slope & $0.323 \pm 0.002 \mathrm{RU} / \mathrm{s} / \mu \mathrm{g} \mathrm{HA} / \mathrm{mL}$ \\
\hline $\mathbf{R}^{2}$ & 0.9998 \\
\hline Limit of Detection (LOD) & $0.03 \mu \mathrm{g} \mathrm{HA} / \mathrm{mL}$ \\
\hline Limit of quantification (LOQ) & $0.10 \mu \mathrm{g} \mathrm{HA} / \mathrm{mL}$ \\
\hline
\end{tabular}

Figure 3 Detection of HA in TIV vaccine. A: Calibration curves for TIV vaccine. B: Sensorgrams obtained following the injection of $\mathrm{H} 1 \mathrm{~N} 1$ and $\mathrm{H} 3 \mathrm{~N} 2$ reagents in the biosensor. C: Analytical parameters determined for TIV vaccine using initial binding rate, $\sigma=0.003 \mathrm{RU} / \mathrm{s}$ $(n=4)$.

These preliminary media measurements being performed, the influenza virus SPRi biosensor was used to characterize bioactive virus from cell-based process. As a matter of fact, when it comes to non-specific signal minimization, serum free Ultra-MDCK is the best option and the evaluation of SPRi virus quantification in cell-based production processes was pursued with this medium. Then, in order to account for the non-specific signal generated by process-derived side products (cell debris and extracellular vesicles mainly), samples from non-infected cell culture $\left(20 \times 10^{6}\right.$ cell/T-175 flask at $\left.\mathrm{t}=0\right)$ were harvested after $48 \mathrm{~h}$ and $96 \mathrm{~h}$ of culture, clarified through $2000 \mathrm{~g}$ centrifugation and injected in the SPRi biosensor. The obtained signals are depicted in Figure 4. As can be seen, when using MDCK as culture medium, a non-negligible increase of the non-specific signal (between 4- and 7-time) in the presence of the cells and their side-products was observed. These background signals, generated by cell culture side products, will be subtracted to all subsequent SPRi measurements as to obtain net signals from cell-based bioprocess virus production.

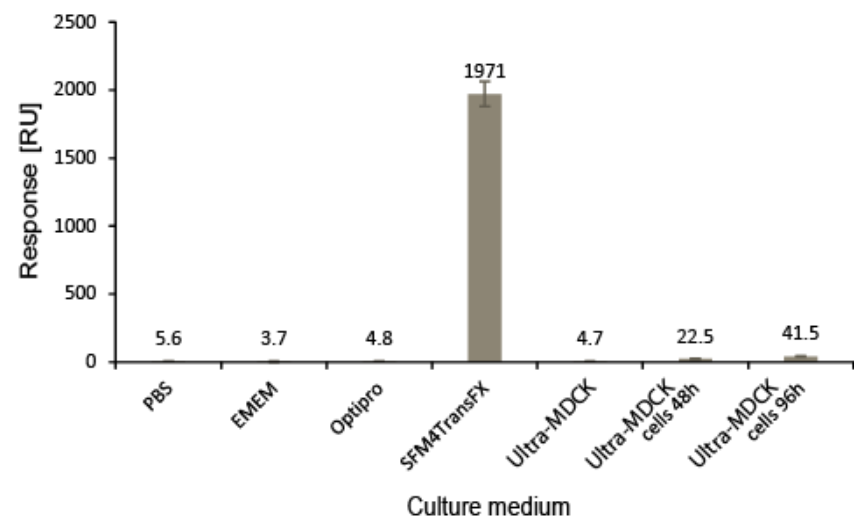

Figure 4 SPRi biosensor responses in the presence of different cell culture media together with SPRi biosensor response of noninfected cell culture harvested after $48 \mathrm{~h}$ and $96 \mathrm{~h}$.

These preliminary measurements being performed, the influenza virus SPRi biosensor was used to characterize bioactive virus from cell-based process. In this case, the sample composition the closest to the production route was used, i.e. $2000 \mathrm{~g}$ clarified cell culture medium. Calibration curve was built toward infectious viral particles since HA concentrations in harvested cell-based culture samples were too low to be determined using SRID method. Results are presented in Figure 5. As presented above, a particular data treatment was applied to these experiments by subtracting sensorgrams from control samples (non-infected culture) to the actual cell-based virus production sample sensorgrams. This treatment was necessary to overcome the matrix effect obtained in the presence of cell culture. As presented in Figure 5-table, the SPRi biosensor allowed to reach a limit of detection of $1.8 \times 10^{5}$ pfu. $\mathrm{ml}^{-1}$ and a limit of quantification of $5.3 \times 10^{5} \mathrm{pfu}^{-1} \mathrm{ml}^{-1}$. The 
linear response was confirmed among a 2 log analytical range, allowing to reach a $10^{7}$ pfu. $\mathrm{ml}^{-1}$ content. Such results allow to envision the monitoring of influenza production in cell-based bioprocess from its early production phases up to its maximal production rates.

These results have to be analyzed taking into account the discrepancy between the mechanisms involved in plaque forming unit (pfu) calculation and the present biosensor. Indeed, pfu assay is impacted only by the presence of infectious viral particles, whereas our fetuin based biosensor quantifies both infectious and non-infectious particles as long as they exhibit active HA, but also cell membrane embedded HA released during cell lysis process.

While infectious viral particles harbor a complete viral genome and are properly conformed for infection, replication and propagation in cell culture, most of the particles generated are defective [11]. Such defective particles were described in numerous previous works as defective interfering particles (DIPs), i.e. particles structurally similar to viruses but partially lacking viral genome [28]. Such DIPs are commonly determined as total viral particles (VP) in production processes monitoring and could be numbered by physical quantification techniques. The ratio between influenza total viral particles (VP) and infectious viral particles (IVP) is commonly evaluated between 10-1000 for influenza A virus, and appeared to vary during cell culture $[11,29,30]$. In the case of the sample used for Figure 5 calibration and harvested $48 \mathrm{H}$ after infection, TRPS quantification of total particles allowed to calculate a VP/IVP ratio of 380 (Supporting Information 3). It is then clear that the SPRi biosensor signal obtained shall be considered only as an indirect detection of IVP (or pfu) through the detection of the total VP concentration. Indeed, only $1 / 380$ of the observed interactions are induced by IVP presence and these interactions correspond to SPRi signals near the apparatus' detection limit (0.1 RU).

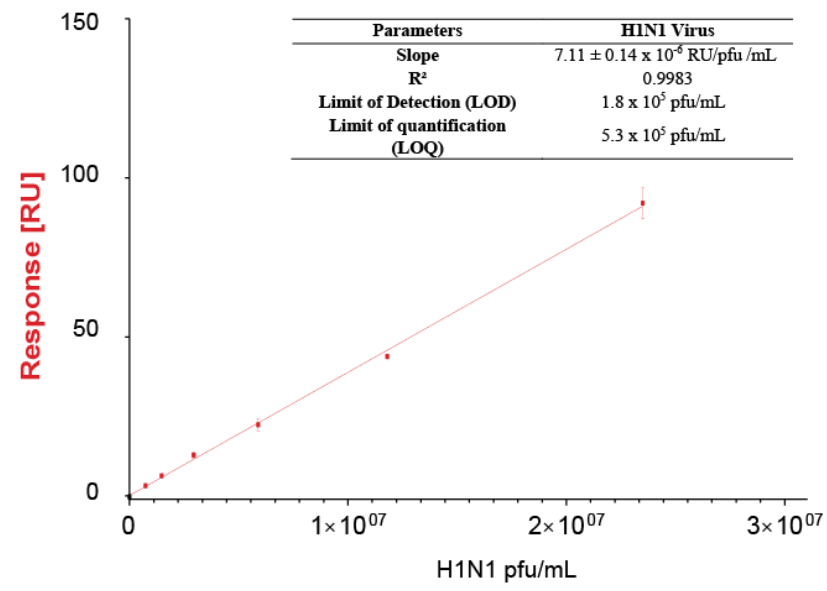

Figure 5 Calibration curve for H1N1 virus from cell-based bioprocess. Insert: Analytical parameters determined for $\mathrm{H} 1 \mathrm{~N} 1$ virus using SPRi response $(n=3)$.

Nevertheless, when it comes to vaccine production bioprocess, VP titer is the primary parameter of interest, as one ultimately wants to evaluate total antigen content in the production process. Indeed, the last set of experiments herein documented is an attempt to demonstrate the capacity of the SPRi biosensor for one-line monitoring of virus particle production. Thus, H1N1-infected cell culture and control cell culture were harvested at regular intervals from 23 to 96 hours postinfection, clarified and injected without any further dilution in the fetuin-based biosensor. For sake of comparison, all cell culture supernatants were also quantified with reference infectivity assays (pfu) and particles counting (TRPS). The experimental results are presented in Figure 6 where each point corresponds to the net signal between infected and control culture. As can be seen, a clear evolution of the biosensor signal was obtained with a SPRi signal leveling off 40 hours after culture infection. This stabilization of the total virus particle content was also observed using TRPS measurements (Supporting Information 3). Figure 6-insert presents the correlation between the measured SPRi signals and the TRPS measurements. A linear correlation $\left(\mathrm{R}^{2}=0.9528\right)$ was found between the two techniques, proof of the usefulness of the developed technique for monitoring vaccine production using cell-based bioprocess.

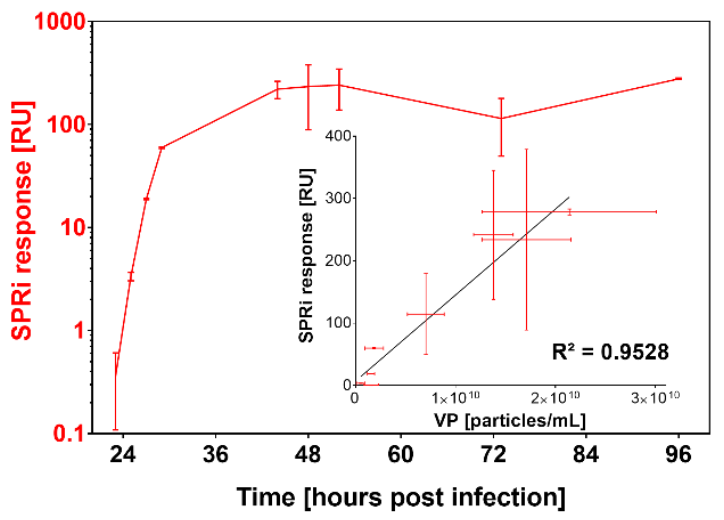

Figure 6 SPRi biosensor signal variations obtained from influenza virus production in MDCK cell-based bioprocess. Insert: correlation between SPRi biosensor response and total viral particles determined using TRPS.

\section{CONCLUSION}

We here presented an assay for the rapid and label-free quantification of influenza virus particles and bioactive hemagglutinin in bioprocess-derived samples, i.e. egg-based and cell-based bioprocesses. The method is based on affinity capture of hemagglutinin antigen by sialic-acid terminated glycans present at the surface of the fetuin-functionalized sensor. Sensor regeneration strategy allows for running multiple sequential analysis on the same sensor, while the use of fetuin glycoprotein provides a panel of sialylated $\alpha-2,3$ and $\alpha$ 2,6 glycans offering specific ligands for influenza HA quantification without discriminating between influenza strains. The assay was shown to offer better analytical performances than SRID or other assays recently developed for influenza vaccine 
evaluation and requires low volume $(<100 \mu \mathrm{L})$ of reagent. The high sensitivity and wide analytical range $(0.03$ and $20 \mu \mathrm{g}$ $\mathrm{HA} / \mathrm{ml}$ ) provide the possibility to evaluate bioprocess samples with low titer. Sample time processing was 10 minutes and is then fully compatible with on-line bioprocess monitoring while SRID turnaround time is usually 2-3 days [1]. The assay was also shown to have the potentiality to be directly applied to cell-based bioprocess monitoring. A minimum sample pretreatment (cell/supernatant separation through $2000 \mathrm{~g}$ centrifugation) was required before analysis, which might in a near future be implemented on-line using membrane and/or acoustic filtration for sample pre-treatment [31].

\section{ASSOCIATED CONTENT}

\section{Supporting Information}

The Supporting Information is available free of charge on the ACS Publications website.

Supporting Information 1 - Sensor regeneration optimization (vaccine $10 \mu \mathrm{g} \mathrm{HA} / \mathrm{mL}$ )

Supporting Information 2 - Comparative evaluation of kinetic constants obtained for reference standards

Supporting Information 3 - Total particles and infectious particles determination in cell-based bioprocess.

\section{AUTHOR INFORMATION}

\section{Corresponding Author}

Univ Lyon, GEMBAS Team, CNRS UMR 5246, INSA, CPELyon, ICBMS, Université Claude Bernard Lyon 1, Villeurbanne cedex, France

Email: christophe.marquette@univ-lyon1.fr

\section{Author Contributions}

The manuscript was written through contributions of all authors. / All authors have given approval to the final version of the manuscript.

\section{ACKNOWLEDGMENT}

L. Durous acknowledges the Auvergne Rhône-Alpes Region for his PhD grant (ARC 1 Santé). The authors have no other relevant affiliations or financial involvement with any organization or entity with a financial interest in or financial conflict with the subject matter or materials discussed in the manuscript.

\section{REFERENCES}

(Word Style "TF_References_Section"). References are placed at the end of the manuscript. Authors are responsible for the accuracy and completeness of all references. Examples of the recommended formats for the various reference types can be found at http://pubs.acs.org/page/4authors/index.html. Detailed information on reference style can be found in The ACS Style Guide, available from Oxford Press.
(1) Thompson, C. M.; Petiot, E.; Lennaertz, A.; Henry, O.; Kamen, A. A. Virology journal 2013, 10, 141.

(2) WHO, Global Influenza Programme. Influenza Vaccine Response during the Start of a Pandemic.: Geneva, 2015.

(3) Minor, P. D. Vaccines 2015, 3, 90-104.

(4) Khurana, S.; King, L. R.; Manischewitz, J.; Coyle, E. M.; Golding, H. Vaccine 2014, 32, 2188-2197.

(5) EMA/EDQM, Joint EMA/EDQM workshop on improved potency assays for inactivated influenza vaccines.

(6) Kuck, L. R.; Saye, S.; Loob, S.; Roth-Eichhorn, S.; ByrneNash, R.; Rowlen, K. L. Vaccine 2017, 35, 1918-1925.

(7) Bodle, J.; Verity, E. E.; Ong, C.; Vandenberg, K.; Shaw, R.; Barr, I. G.; Rockman, S. Influenza and other respiratory viruses $\mathbf{2 0 1 3}$, 7, 191-200.

(8) Buffin, S.; Ikhelef, N.; Prudent, J.; Dubayle, J.; Nougarede, N.; Varenne, M. P.; Moste, C.; Legastelois, I. J Virol Methods 2018, 251, 46-53.

(9) Pierce, C. L.; Williams, T. L.; Moura, H.; Pirkle, J. L.; Cox, N. J.; Stevens, J.; Donis, R. O.; Barr, J. R. Anal Chem 2011, 83, 47294737.

(10) Wen, Y.; Han, L.; Palladino, G.; Ferrari, A.; Xie, Y.; Carfi, A.; Dormitzer, P. R.; Settembre, E. C. Vaccine 2015, 33, 5342-5349.

(11) Heider, S.; Metzner, C. Virology 2014, 462-463, 199-206.

(12) Jacquemart, R.; Chavane, N.; Durocher, Y.; Hoemann, C.; De Crescenzo, G.; Jolicoeur, M. Biotechnol Bioeng 2008, 100, 184-188.

(13) Nilsson, C. E.; Abbas, S.; Bennemo, M.; Larsson, A.; Hamalainen, M. D.; Frostell-Karlsson, A. Vaccine 2010, 28, 759-766.

(14) Erickson, H. P. Biol Proced Online 2009, 11, 32-51.

(15) Schasfoort, R. B. M.; Royal Society of Chemistry: Cambridge, pp 60-105.

(16) Kon, T. C.; Onu, A.; Berbecila, L.; Lupulescu, E.; Ghiorgisor, A.; Kersten, G. F.; Cui, Y. Q.; Amorij, J. P.; Van der Pol, L. PLoS One 2016, 11, e0150700.

(17) Wood, J.; Schild, G.; Newman, R.; Seagroatt, V. Jounal of Biological Standardization 1977, 5, 237-247.

(18) Jiang, L.; Eichelberger, M. C. Biol Proced Online 2015, 17, 7.

(19) Khurana, S.; Verma, S.; Verma, N.; Crevar, C. J.; Carter, D. M.; Manischewitz, J.; King, L. R.; Ross, T. M.; Golding, H. PLoS One 2010, 5, e11548.

(20) Takemoto, D. K.; Skehel, J. J.; Wiley, D. C. Virology 1996, 217, 452-458.

(21) Carvalho, S. B.; Moleirinho, M. G.; Wheatley, D.; Welsh, J.; Gantier, R.; Alves, P. M.; Peixoto, C.; Carrondo, M. J. T. Biotechnol J 2017, 12 .

(22) Magadan, J. G.; Khurana, S.; Das, S. R.; Frank, G. M.; Stevens, J.; Golding, H.; Bennink, J. R.; Yewdell, J. W. J Virol 2013 , $87,9742-9753$

(23) Healthcare, G.

(24) Gerlach, J. Q.; Kilcoyne, M.; Joshi, L. Anal. Methods 2014, 6, 440-449.

(25) Kuck, L. R.; Sorensen, M.; Matthews, E.; Srivastava, I.; Cox, M. M.; Rowlen, K. L. PLoS One 2014, 9, e109616.

(26) 2008:., C. o. E. E. P., INFLUENZA VACCINE (SPLIT VIRION , INACTIVATED); 2008.

(27) Lohr, V.; Rath, A.; Genzel, Y.; Jordan, I.; Sandig, V.; Reichl, U. Vaccine 2009, 27, 4975-4982.

(28) Milián, E.; Julien, T.; Biaggio, R.; Venereo-Sanchez, A.; Montes, J.; Manceur, A. P.; Ansorge, S.; Petiot, E.; Rosa-Calatrava, M.; Kamen, A. Vaccine 2017, 35, 3423-3430.

\section{TABLE OF CONTENT}




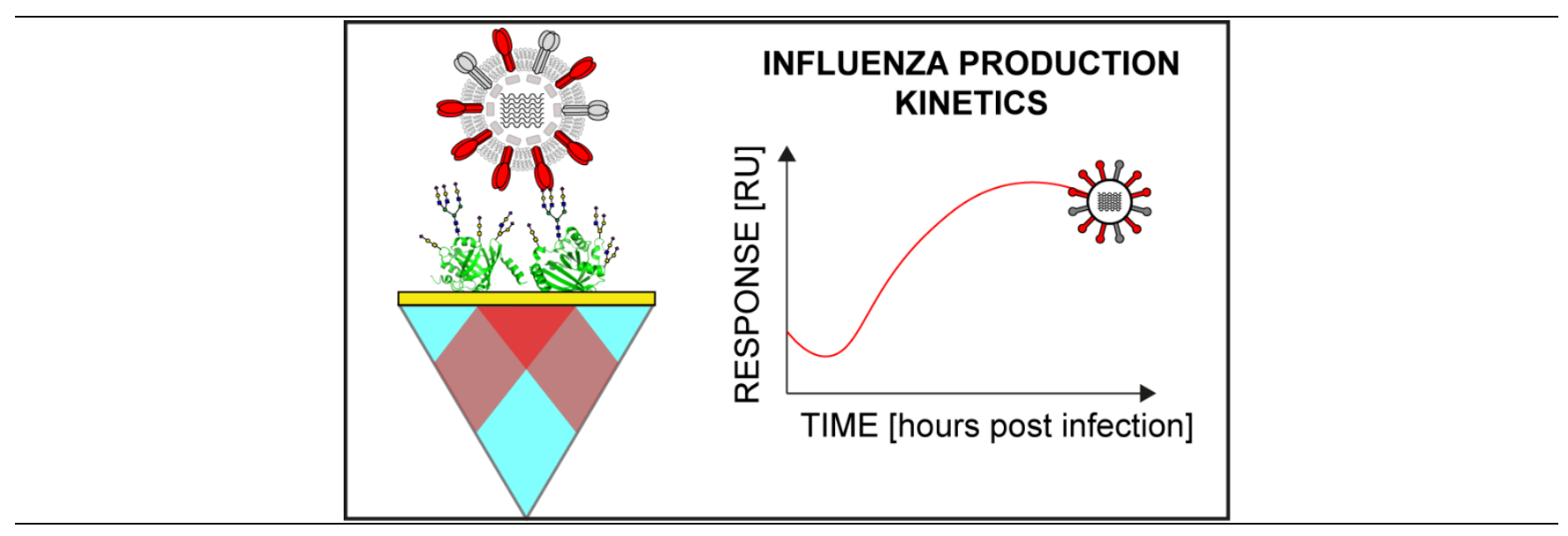

